\section{General relativity}

Gravitation. By C. W. Misner, K. S. Thorne, and J. A. Wheeler. Pp. xxvi+ 1279. (Freeman: San Fransisco and Reading, December 1973.) $£ 19.20$ boards; $£ 8.60$ paper.

IN most cases it is probable that, if a scientific discovery had not been made by the person normally credited with it, it would have been made by someone else within a few years. There is one notable exception to this general rule: had Einstein died before 1916, the world would have probably had to wait another 50 years or so for the General Theory of Relativity. In fact Einstein was ahead of his time: after an initial burst of activity in the $1920 \mathrm{~s}$, there was very little work on the theory until about 1960 . This neglect was caused partly by the belief that the theory was too complicated to allow predictions to be made in any but the simplest cases and partly by the fact that there did not seem any prospect of comparing the predictions with observation apart from the three famous Solar System tests which Einstein himself proposed.

During the period 1920-1960 the most exciting field of physics seemed to be quantum theory. The success of quantum electrodynamics led most physicists to feel that physics was Feynman diagrams. They distrusted general relativity because of its classical and geometric nature. This dislike of geometry is shown, for example, by the particle physicist Steven Weinberg in the introduction to his otherwise excellent book Gravitation and Cosmology (John Wiley, New York, 1972): “. . . too great an emphasis on geometry can only obscure the deep conncetions between gravitation and the rest of physics. .... I believe that the geometrical approach has driven a wedge between general relativity and the theory of elementary particles".

But geometric insights have played an important part in many of the outstanding recent theoretical developments such as black holes, space-time singularities and gravitational radiation. The answer therefore seems to be not to free general relativity from geometry but to teach geometry to physicists brought up on the normal ideas of special relativity; that is, to teach them to regard space-time as a differentiable manifold and not as a flat vector space.

This is what Misner, Thorne and
Wheeler have set out to do in their book. They have also endeavoured to give at least an introduction to those fields such as cosmology, gravitational waves, post-Newtonian effects in the Solar System, relativistic stars and black holes where general relativity is now, or is on the point of, making contact with the recent great advances in observation. All this combined with fairly large print and wide margins has produced a book weighing 6 pounds. Because this is too long for most postgraduate courses on general relativity, the authors have divided the material into two tracks which are indicated on the top corners of the pages. Track 1, which is designed to accompany a one-semester course, gives an impressionistic overview of the theory and its applications but to perform any calculation one would really need to master most of the Track 2 sections on the basic theory.

The style is typical of the Princeton school (Misner and Thorne are former students of Wheeler at Princeton). It is aimed more at giving the reader an intuitive feeling for the concepts involved than giving a mathematically rigorous axiomatic account. Important results and other material of interest are displayed in boxes. These are generally helpful though they sometimes rather interrupt the text. The book is also well provided with exercises ranging from easy to major research problems. These should be very helpful to students learning the subject.

The authors' research interests have obviously influenced the coverage of the book. There are very good sections on relativistic stars, black holes and experimental tests of relativity. On the other hand the section on cosmology is less detailed than that in Weinberg's book and suffers from the ideological insistence that the Universe is spatially closed. The section on gravitational waves gives a good account of the generation and detection of gravitational radiation but does not mention the work of Bondi, Sachs, Newman and Penrose on the 'peeling off' of the curvature tensor, the Bondi mass loss formula and the Bondi-MetznerSachs group. These and the comparative neglect of spinors are the main gaps in this book, which does not aim to describe in detail global results such as singularity theorems. Despite these omissions the book is a major landmark in the field and is likely to become the most widely used textbook and reference work on the subject. It is well printed and bound and the paperback version is cheap for a book of this size.

S. W. HAWKING

\section{Radial parameters}

Ligand-field Parameters. By M. Gerloch and R. C. Slade. Ppxii +235. (Cambridge University: Lo ndo n, August 1973.) £5; \$15.

Although many books exist dealing with the theory of transition-metal complexes, this one is welcome, since it treats the subject in a novel way. Other texts approach ligand-field theory through symmetry and group theory, being concerned with aspects of the subject determined by molecular symmetry alone. This is a valid approach, but it is sometimes necessary to stress those parts of ligand-field theory where the molecular symmetry is less significant, and this is done here.

For all systems, group theory can only give information on quantities dependent upon the angular properties of wave functions. Radial properties appear only as proportionality constants. Furthermore, complexes of lower than cubic symmetry are less amenable to group-theoretical treatment, needing large numbers of parameters to describe the system.

In this book stress is laid on calculations of radial properties of regular octahedral and tetrahedral complexes, and the ligand-field parameters of noncubic systems, thus rationalising pertinent data on such complexes. A summary of the role of symmetry in ligandfield theory is followed by a development of the crystal-field formalism, with a discussion of inter-electron repulsion parameters in cubic systems. Treatment of the radial parameters in various complexes of lower symmetry follows, with emphasis on trends in the parameters $C p$ and $D q$.

The second half of the book seeks to interpret these radial parameters, covering calculations of $10 \mathrm{Dq}$, and use of the point charge model and semiempirical molecular orbital methods (especially the angular overlap model) in rationalising trends in $D q$ and $C p$. The final chapter contains a discussion of the Nephelauxetic effect.

The authors hope that the book will be useful to advanced undergraduates and young researchers; but although the treatment is sound and logical, the level of difficulty will probably prove to be too great for all save very few 